\title{
One year of Nature Cancer
}

\author{
This month marks one year since the launch of Nature Cancer. As we celebrate our first anniversary, we reflect on \\ the past year and thank the cancer research community for embracing our journal.
}

I I 1 ay you live in interesting times" - so says an old curse. When we were launching Nature Cancer last January, nothing could have prepared us for quite how interesting 2020 would turn out to be. A year in which superlatives were used in a literal sense, 2020 was truly extraordinary and, for too many people, an annus horribilis. As the COVID19 pandemic tightened its grip around the globe, human suffering and loss of life continued to rise, leading to widespread lockdowns, triggering a deep global financial crisis and largely upending day-to-day life. The necessary focus on COVID-19 impacted the provision of non-COVID-19-related healthcare for many patients. Research efforts that were unrelated to the pandemic at times paused completely or continued at a slower pace, in line with lockdown mandates. Many scientists saw their career plans derailed due to funding and hiring freezes. Apart from the pandemic, the world experienced political and social upheaval, a time of racial reckoning and renewed urgent calls for equity, diversity and inclusion.

However, 2020 was also a year during which science and medicine shone, with healthcare workers and researchers in academia and the industry working tirelessly to help patients and develop therapies and vaccines.

Scientific publishing, including the Nature journals and Nature Cancer, was not unaffected. Journal editors and production teams strove to ensure that COVID-

19-related research was prioritized while handling increased manuscript volumes and addressing the disruptions the pandemic caused on a professional and personal level, not only for many authors and referees but also for journal staff. And yet, all challenges aside, 2020 was exciting for Nature Cancer, and not simply because it was the journal's first year of existence. Of course the success of a journal cannot be assessed in 12 short months, but with this whirlwind year behind us, now is a good opportunity to take stock of what we have achieved so far and what lies ahead for 2021 and beyond.

For Nature Cancer, 2020 had an auspicious start with a first issue that captured the diversity of topics and content we intend to offer and was received enthusiastically by the cancer community. In our first Editorial ${ }^{1}$, we outlined our aims to serve the wider cancer field, to foster the exchange of innovative ideas and to provide a platform for the convening of researchers across the disciplines addressing the global challenge of cancer. In line with this goal, in our first 12 issues we published primary research papers across the spectrum of preclinical, translational and clinical research, as well as Review, News and Comment articles providing rich discussion and diverse opinions on biomedical, policy and societal aspects of the cancer field. To celebrate the milestone of one year of Nature Cancer, we present a specially curated anniversary collection of research papers published in cancer biology, cancer genomics, and translational and clinical research - three major and broad areas of the journal's scope - with a few highlights of comment and opinion pieces.

In the field of cancer biology, we have published a wide variety of papers addressing the molecular and cellular underpinnings of cancer development and metastasis, including studies focusing on the interplay of cancer and metabolism. The complex inputs of the tumor microenvironment, the immune system and the systemic effects associated with cancer also featured prominently in our first-year content.

In cancer genomics, primary research included studies on the impact of cancer genomes, epigenomes and gene-expression programs on tumor development, progression and response to therapy. We also published papers developing and employing innovative technologies and computational tools, including through artificial intelligence approaches, to profile and stratify tumors and identify cancer biomarkers and therapeutic targets. We further highlighted the impressive progress in this field through a Series on Clinical Cancer Genomics featuring specially commissioned Review and Perspective articles, and produced two webcasts in which experts discussed technological advances and clinical applications of next-generation sequencing. In 2021 and the coming years, we plan to highlight developments in other major areas of cancer research through similar projects.

Our publications in the translational and clinical space ranged from preclinical, exploratory work on testing therapeutic targets and identifying drugs and treatment combinations, to clinical trials. Of particular note was research on the development and testing of immunotherapy modalities, including by engineering immunomodulatory antibodies, cell-based therapies and cancer vaccines. To expand our coverage of clinical oncology, in this issue of Nature Cancer we are introducing a new article type called 'Clinical Outlook ${ }^{2,3}$. This new format aims to provide timely, focused and high-quality discussions on the latest clinical progress in a specific area of oncology, written by experts in that particular field. In the coming months, we plan to publish a number of such pieces, covering ongoing or recent clinical trials and other notable clinical developments for a particular cancer type and various treatment and diagnostic modalities or molecular targets.

COVID-19 could not be absent from our pages, and although its interplay with cancer is still emerging, we approached the topic not only through primary research publications on the effect of SARS-CoV-2 infection on the risk and outcomes of patients with cancer, but also through Editorials and Comment and Review articles on the influence of COVID-19 on the cancer field, such as in the context of immuno-oncology, cancer care and clinical trial design.

Finally, we published Comment, Review and News articles on the impact of cancer on society, particularly on health disparities and the financial burden of this disease. These are areas on which we hope to expound with time, with more commissioned and research articles and a broader focus on disparities and diversity.

Indeed, in a year during which life as we knew it changed dramatically, many of our priorities had to be reevaluated and plans had to be postponed, not only regarding content but also community engagement. One of the things we missed most was meeting researchers at conferences and institute visits, and although we made the most of virtual meetings, we eagerly await the time when we can travel and interact in person safely. In fact, as of this writing, the Nature Cancer team members, all of whom are based in New York and London, continue to work exclusively from home, and have done so since last March. Apart from the team of dedicated professional 
editors who handle all content from submission to publication, Nature Cancer includes production, administrative and publishing teams without whom it would not be possible for the journal to operate. We thank the proverbial village of colleagues whose hard work makes Nature Cancer what it is and are grateful that we have been able to continue working and publishing throughout the year despite the challenges we have faced.

To our authors who have entrusted their work to us, to our referees who, through their expertise and time, help us provide a rigorous and constructive peer-review process, and to our readers who embraced our journal so warmly from its very early days, we extend our deepest gratitude. We renew our commitment to serve the cancer community with integrity, striving to offer a fair and efficient editorial and peer-review experience and to publish impactful research of the highest quality. Journals are shaped by the vision of their editors and the needs and input of the communities they inform. As Nature Cancer continues to grow, we look forward to engaging more deeply with the cancer community, and we always welcome your feedback at cancer@nature.com or by contacting individual editors.

Published online: 13 January 2021

https://doi.org/10.1038/s43018-020-00170-9

References

1. Nat. Cancer 1, 1-2 (2020).

2. DiNardo, C. D. \& Konopleva, M. Y. Nat. Cancer https://doi org/10.1038/s43018-020-00165-6 (2020).

3. McMullen, M., Karakasis, K., Rottapel, R. \& Oza, A. M. https://doi.org/10.1038/s43018-020-00166-5 (2020). 